\title{
15
}

\section{A signaling based approach to broadband service control. Application to access signaling}

\section{P. Martins}

Ecole Nationale Supérieure des Télécommunications, Paris, France. e-mail: martins@res.enst.fr

\section{Rigault}

Ecole Nationale Supérieure des Télécommunications, Paris, France. e-mail: rigault@res.enst.fr

\section{N. Raguideau ${ }^{\prime}$}

Telecommunication systems Business Unit, Hewlett Packard, Grenoble, France.

e-mail : Nicolas_Raguideau@grenoble.hp.com

\begin{abstract}
This paper describes a new signaling based approach for the implementation of a control plane for the B-ISDN network. A control architecture is developed, with a separation between service session, access session and call. This architecture provides the guidelines for the definition of signaling protocols. The differences between this architecture and other existing control plane proposals are reviewed. The emphasis is put mostly on the access session signaling and the physical architecture. However some indications on the service control signaling are given.
\end{abstract}

\section{Keywords}

B-ISDN signaling, Control plane, Call Model, Session concept

\footnotetext{
' The ideas presented in this paper represent the author's point of view and not necessarily the point of view of their respective institutions. 


\section{INTRODUCTION}

The purpose of this paper is to define suitable signaling schemes for the control plane of the broadband network. We start from a control architecture compatible with already pending proposals such as B-ISDN Capability Set 3 (B-ISDN CS3), IN Capability Set 3 (IN-CS3), and TINA-C (Darmois, 1996) (Barr, Boyd, Inoue, 1993). However our scheme differs from the above mentioned architectures by the importance given to signaling, both for the service session and the call session. Differences with other approaches will be underlined as we explain the architecture. The model will then be used to develop the access signaling and to introduce service session signaling on a service example.

\section{THE PROPOSED ARCHITECTURE COMPARED TO PREVIOUS APPROACHES}

The proposed architecture has been described in details (Rigault, Kovacikova 1997). It is based on the TINA-C view of the telecommunication business model (Figure 1). This model is characterized by five business roles: the "consumer" who asks for services and gets billed for it, the "retailer" providing access to the services and reselling to the consumer telecom usage bought in bulk at bargain prices to a "connectivity provider", the "third party service" providers using the broadband network to provide an infinite variety of services, and the "broker" used to find out which services are provided and their identity.

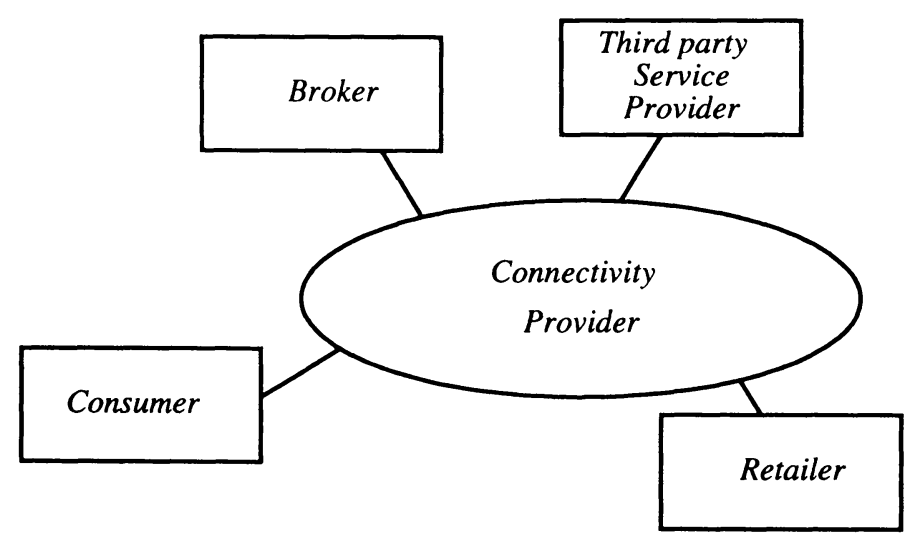

Figure 1 Business roles in the B-ISDN network.

Before B-ISDN CS3, the concept of a call was equivalent to the negotiation and allocation of communication resources between users, independently of service specific functionalities, and user roles in the service. Such a call concept was 
mixing in the same process the logical establishment of the association between parties, the search for network resources and the actual connection of the bearer facilities between these resources and parties. In order to solve the problems of the variety of connectivity requirements both in topology and set up sequences for the expected new services, we adopt the clear separation introduced by B-ISDN CS3 between the session concept and the call concept (Figure 2).

The Service Session in B-ISDN CS3 is a single instance of service use with global significance. It includes the end to end view between user applications, while the call includes the end to end view between network access points. The Service Session takes place in the service provider platform (eventually distributed over several servers linked by networks). The Service Session takes care of service parameters like coding schemes, but does not take care of network aspects like QOS parameters. On the other hand the call takes place in the network (or the networks) involved by the service. In its new meaning, the call represents an association of one or more parties, using a telecommunication service to communicate through the network. The correspondence between session and calls may be of the "one to many" type, since a given Service Session may involve several calls or no calls at all. A session may be kept set up, while the corresponding call is released for some time. Upon resumption of the session activity a new call will be set up.

The Service-Call separation is a necessity for services to be provided by other stake holders than the network operators.

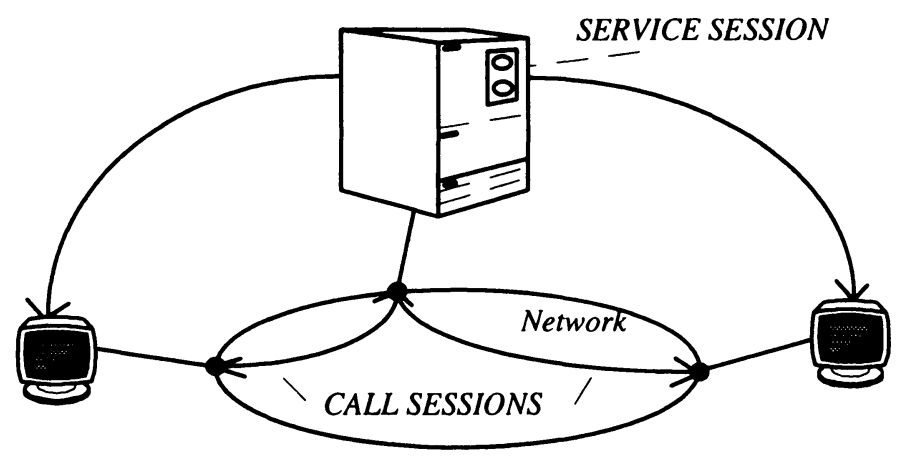

Figure 2 Service Session and calls in the B-ISDN network.

Due to mobility, it will be very frequent that the user of a given terminal will not be it's owner. However each user will have unique characteristics such as environment preferences, personal subscriptions and so on. It is therefore advisable, following again the TINA approach, to provide an Access Session. The access session brings the user profile down to the actual access switch of the terminal presently used by the consumer. More generally, it takes care of customer related functionalities, like the Home Location Register (HLR) of mobile telephony does it today, and presents 
a generic interface to the Service Session. The Access Session is executed on the platform of the retailer providing telecommunication services to this consumer.

All above considerations finally lead to the control plane functional architecture shown in Figure 3. This architecture entails the following functional entities.

- Terminal Manager Function (TMF) : terminal related, provided by the operating company supplying connection services to the terminal in use. The TMF is a generalization of the role played by the Visitor's Location Register (VLR) of mobile telephone networks. It gets from the access function the user profile of the consumers using its related terminals.

- Access Session Function (ASF) : customer related, provided by a retailer type of business which may also be an operating company. The ASF is a generalization of the role played by the Home Location Register (HLR) of mobile telephone networks. It keeps information on the exact status of all consumers associated with this access provider.

- Service Session Control Function (SSCF) : third party service supplier related or operating company related. The switches will normally contain the SSCF for basic services

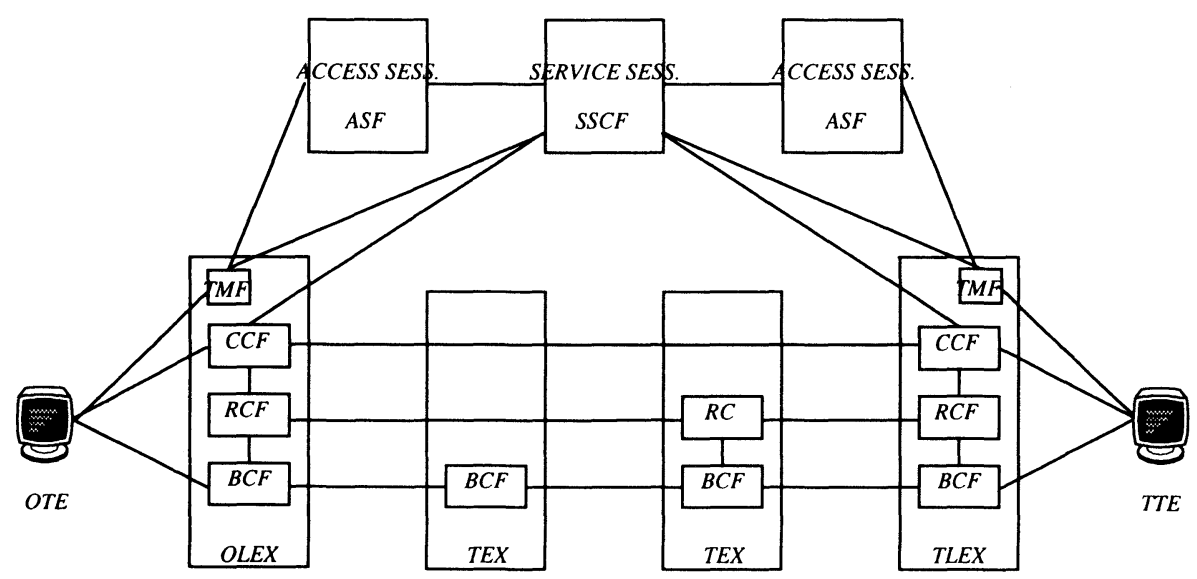

Figure 3 Control plane Functional Architecture.

To underline the differences between this architecture and Intelligent Network (IN) solutions we remark that IN uses the call as a device to trigger a service instance, giving therefore precedence of the call over the Service Session. The main issue then, is of the feasibility of an "universal, any service call model". Could any type of service be triggered from a call ? This idea is very much challenged and some services are known today as "call unrelated" services (such as updating the location of a mobile user, a short message service, etc.). Actually services constitute an open set. It is likely that new services that cannot be triggered from the current call model will always be proposed. We therefore suggest the service precedence 
principle according to which the service should be triggered first, and the call, if any call is required, should be triggered afterwards. Only in this manner generic call procedures may be designed. This service precedence is not found in IN architectures or in B-ISDN architectures. It is however found in the TINA-C proposal.

The first key point of our control architecture lies therefore in the provision of a direct signaling path between the Consumer and the Service Provider platform to initiate the service session first. If required, the call or the calls (some services may require several calls in different networks) may then be originated, either by an instruction of the service instance to the originating customer equipment, or as a direct third party connection request to the network.

A second key point in this architecture concerns the nature of the communication paths between the functional entities. These paths are direct signaling paths and do not require the use of a middleware like the Distributed Programming Environment (DPE) of TINA-C, making there an important difference between our architecture and the TINA-C proposal. While we use the object oriented technology for design, we prefer not to resort to the DPE for the implementation.

Indeed, the important objectives of our architecture is compatibility with legacy system, sufficient control of network parameters from the service application and performance regarding service and call setup times. Parties should not experience significant delays after joining agreement, mostly if these parties are information servers in the case of information retrieval services.

Due to these performance considerations, a DPE should be considered as a fast real time system. In particular, one of the issues to be considered for a DPE is the implementation of efficient naming / trading services for telecommunications. An other issue is to be found when taking in account high availability aspects in distributed object oriented architectures where the semantic attached to certain information elements like object references needs to be revisited for keeping performance. Regarding such issues, and others, it is generally agreed that the achievement of a performant real time DPE, able to interwork with a sizable amount of distributed objects is not to be available in a near future. As an example of such concerns, the CORBA environment presently considered as the best candidate for the DPE does not provide mechanisms for end to end QOS definition, does not provide a priority system for requests and does not provide a blocking protection when serving the requests (Schmidt 1997).

We therefore propose an architecture that is compatible with the service objectives of TINA-C and which is implementable as an evolution of legacy systems without having to wait for the availability of an adapted DPE environment. Furthermore our architecture, by its compatibility, does not prevent the development of distributed middleware when the technology will become available. In the meanwhile, our proposed architecture is adapted to favor fast service setup times.

An other point to be underlined is the compatibility of this architecture with Connectionless and Connection oriented Services. The SSCF has two different ways to establish a call. In a first method, the called party address is loaded into the 
user's Originating Terminal Equipment (OTE). A connectionless call may then be initiated from this OTE.

A connection oriented call may also be originated in this manner via signaling from the OTE. However, it seems more advantageous to initiate connection oriented calls via a third party connection request sent by the service session to the call control function of the OLEX.

The call may now proceed by a Call Control instance, including look ahead procedure to know if the terminals are able to accept the call and to negotiate the QOS, resource control is activated to locate suitable network resources, and bearer control is finally performed to set up all the bearer connections.

\section{ACCESS NETWORKS PHYSICAL ARCHITECTURE}

The access network configuration in the new telecommunication infrastructures will be based on SDH loops connecting remote Broadband concentrators to the connectivity provider switches (Figure 4). Some cooperation has to take place between connectivity providers to share access to given SDH loops, and broadband concentrators must have enough switching abilities to direct calls to one operator switch or to an other since it is expected in the future that the user will be allowed to select his connectivity provider.

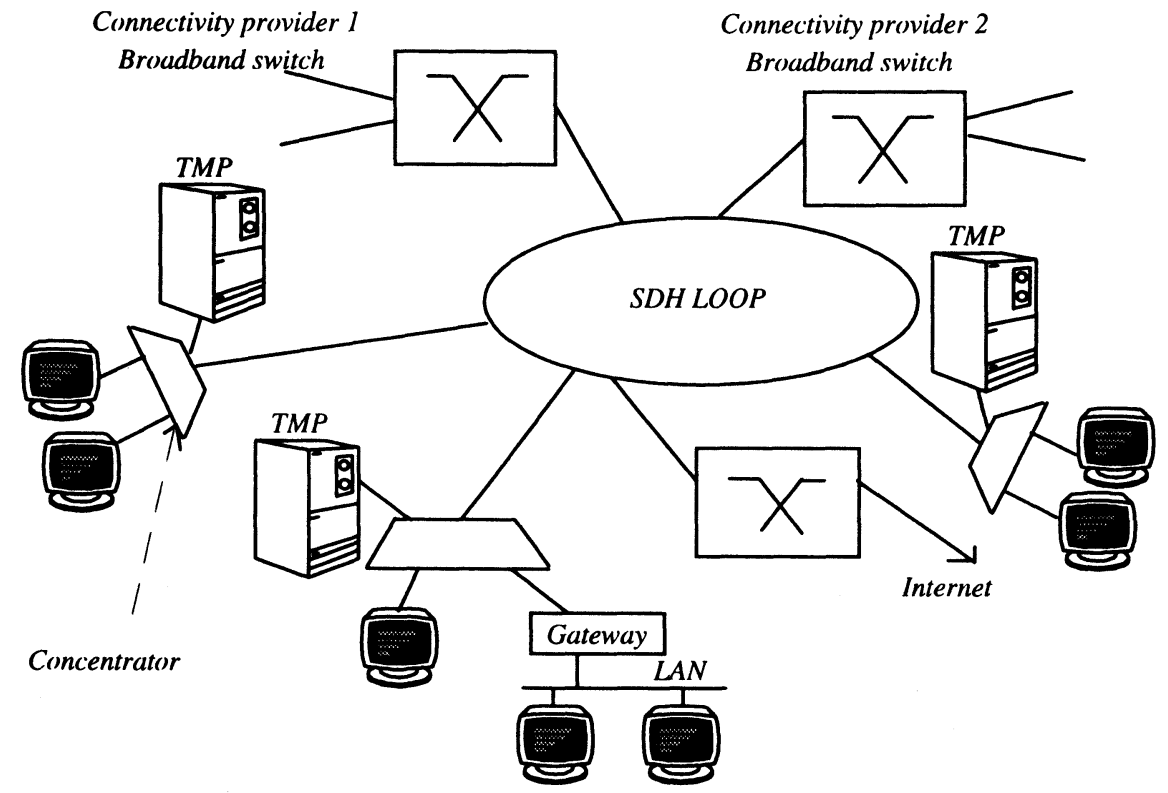

Figure 4 TMP and the access networks. 
The TMP is the control interface between the user and the access and service session. Concentrators and TMPs are operator equipment. The TMP should be placed behind the concentrators so that each terminal gets a full availability access to it. In addition such a location at some operator site is required given its role in critical processes like billing and user's authentication. Whenever a user initiates a service session, the service provider, via service session signaling messages (between SSCP and TMP), informs the user of which connectivity providers he can access. The user selects on his screen, a connectivity provider, and this choice is sent in a signaling message. The TMP will redirect the chosen connectivity provider identity to the call control function of the concentrator. For terminals directly connected in ATM to the concentrators, a connectionless signaling path is established between the terminal and the TMP.

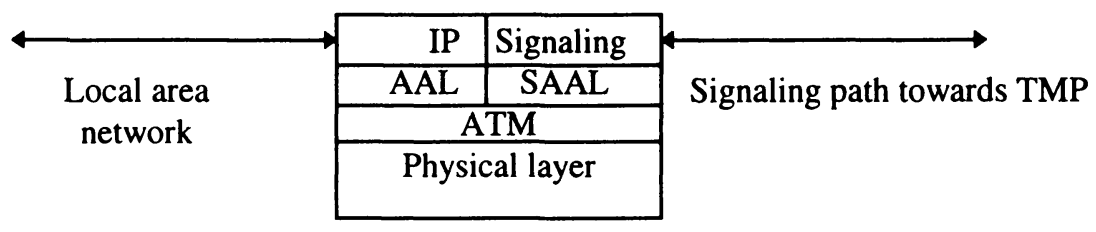

Figure 5 A gateway.

However terminals may be connected via some Local Area Networks not necessarily based on ATM technology. In such a case, a new gateway equipment (Figure 5) providing interworking functionalities should be provided to interface with the ATM network. Such an approach for a gateway in a local area network based on TCP/IP stack is used in the ITU-T H.323 gateway recommendation (H.323). In this case, a connectionless signaling path is established between the gateway and the TMP.

\section{ACCESS SESSION SIGNALING}

In order to describe how a service session is activated and calls are set up in our architecture, we must first define the concepts of "user profile" and "login file".

User Profile: We call "user profile" a set of information characterizing a particular consumer that are necessary to be known in order to establish telecommunication services for this consumer. Examples of such information are :

- his authentication information

- his preferred graphic environment (including wallpaper, colors, screen saver...)

- pointers for his various mail services (email, voice mails...)

- his bookmarks

- his applications

- his billing records 
- his subscriptions in the case of non generally available services

- his list of terminals associated to his eventual "one number" service. (a list of terminals where he is likely to be joined)

It is the responsibility of the retailer or access provider to keep the user profile available and updated in the Access Session Function. A copy of this user profile is sent to the Terminal Manager Function of the switch hosting the terminal that is currently used by this consumer.

Login file: We call "login file" an information element sent by means of signaling by the terminal to Terminal Manager Function which contains among others :

- the identity of the calling consumer (unrelated to the terminal).

- the identity of the retailer or access session provider for this consumer

This login file may be memorized on various media, such as a SIM card or any other portable memory device, that would be generally accepted by all terminal equipment.

The Economy Principle: We shall use consistently this principle according to which no signaling process should be made systematic if not strictly required. As an example, if the user profile is already cached in the Terminal Manager Function (like it will be in most cases), no signaling should take place to the Access Session Function.

Having defined important datas regarding the consumer, we will now describe the exchange of signaling messages by which the access session may develop by using these data.

\section{Originating access session sequences}

The user initiates the access session by placing his Login file storage medium in a terminal. The access session sequence entails the authentication of the user followed by the downloading of the user profile into the TMP (in case it is not already cached there).

Signaling steps (Figure 6) are the following :

- A login message is sent to the TMP. It contains the International Terminal Equipment Identity ITEI of the Originating Terminal Equipment OTE and the International Subscriber identity ISI of the person presently using the OTE. The ISI points to the ASP and to the name of the subscriber in the ASP. The TMP checks if the user's profile is in its cache. If it is the case, an authentication procedure similar to the MAP authentication, is performed and a Login_ack message, containing the calling user's profile (UPF) is returned to the OTE. Otherwise, a send_authentication_info request is sent to the ASP. After completion of the authentication procedure the TMP sends a Send_user_profile message to the ASP to request the user's profile. 
- The ASP returns the user profile to the TMP in a Send_user_profile_ack message. The user profile is then loaded in the terminal. The information elements received are the mail pointers (M_P), the service pointers (SS_P for subscribed service and US_P for unsubscribed services), the user's environment (ENV) and the broker pointers (B_P). The user may now invoke a service.

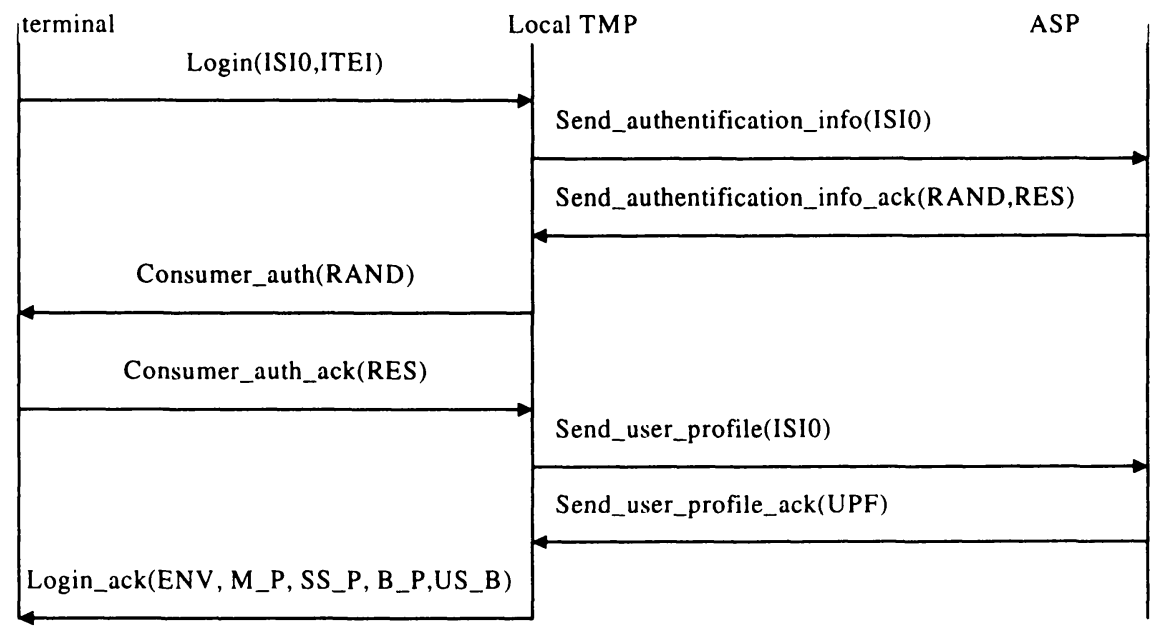

Figure 6 Authentication and user's profile retrieval procedures

\section{Terminating access session sequences}

We now assume that a service session has been initiated in an SSCP, and that a called terminal has to be invited to join in the service session. The SSCP contacts the called party's ASP. This ASP responds with an Terminal_info_type message (Figure 7) containing the called user's network location and the features of the terminal he is presently using. If the type of terminal is compatible with the service logic, the SSCP sends a Terminal_info_type_ack. Then the called ASP contacts the TMP where the called user is located with a terminal_notification message. The TMP notifies the terminal of an incoming service session with a Consumer_notification. The terminal acknowledges the service session notification via a Consumer_notification_ack message. If the called user accepts to join the service session, an Accept_service_session message is sent to the TMP. Then the TMP initiates an authentication procedure such as described previously. After that, the TMP retrieves the user profile from the ASP and acknowledges the request for service session with an Accept_service_session_ack. Finally, the TMP sends a Terminal_notification message to the ASP, and the ASP acknowledges the join service session message. 


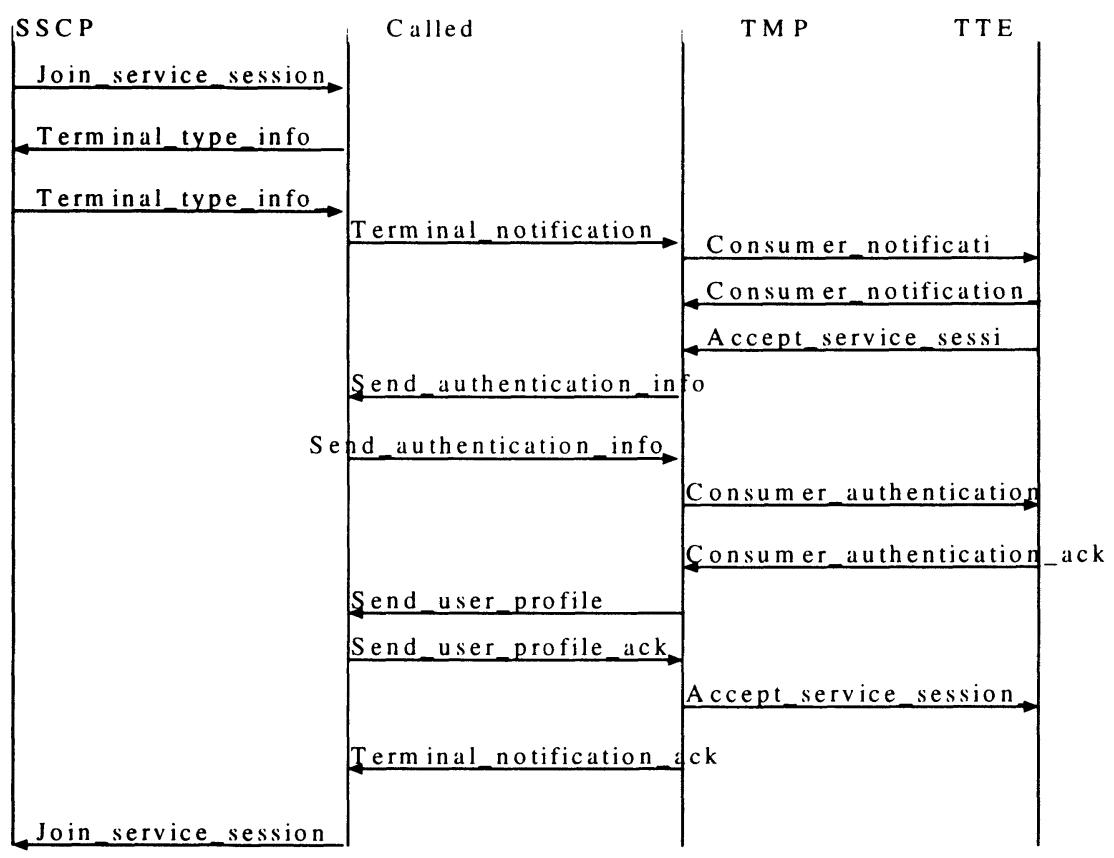

Figure 7 Terminating access session procedures

\section{GENERAL OUTLINE OF SERVICE SESSION SETUP}

The service invocation may proceed according to the following steps (Figure 8). The user clicks on a subscribed service bookmark and a subscribe_service_invoke is directed to the TMP. This message contains the Subscribed service Pointer SS_P which makes it possible to retrieve the network address of the service provider SSCP. Then a Send_user_status is sent to the SSCP containing the user identity and the access provider identity.The SSCP uses these identities to verify the subscription status of the invoking user and access provider. If the status allows, a service session may be instanced and a Send_user_status_ack is returned to the TMP containing service availability, cost, terminal requirements, eventually identity of other parties already involved in the service. These information are forwarded to the user by a Subscribed_service_invoke_ack message. The user may then confirm his service request with a service_confirm message. This message is relayed by the TMP to the SSCP. The SSCP initiates the service session and interacts with the invoking user to acquire all necessary service instance data such as Identities of other parties to be joined in the service, user languages... When all parties have agreed to join in the service, a Service_confirm_ack message is returned to the TMP indicating that the service session is setup. However in most cases calls will have to be setup. The Service_confirm_ack message will therefore 
indicate the calls that have to be initiated, the called parties identities along with the connectivity providers available for each of these parties (CP_P or connectivity providers pointers), and the suitable connecting mode for this service i.e. connection oriented or connectionless. This message is forwarded to the user. The user makes his choices for the current service session, and these options are sent in a Call_Request message to the TMP. The connectionless calls are directly initiated from the TMP. Connection oriented call_request messages are relayed to the SCCP. The SSCP can now contact the connectivity provider to request call setup.

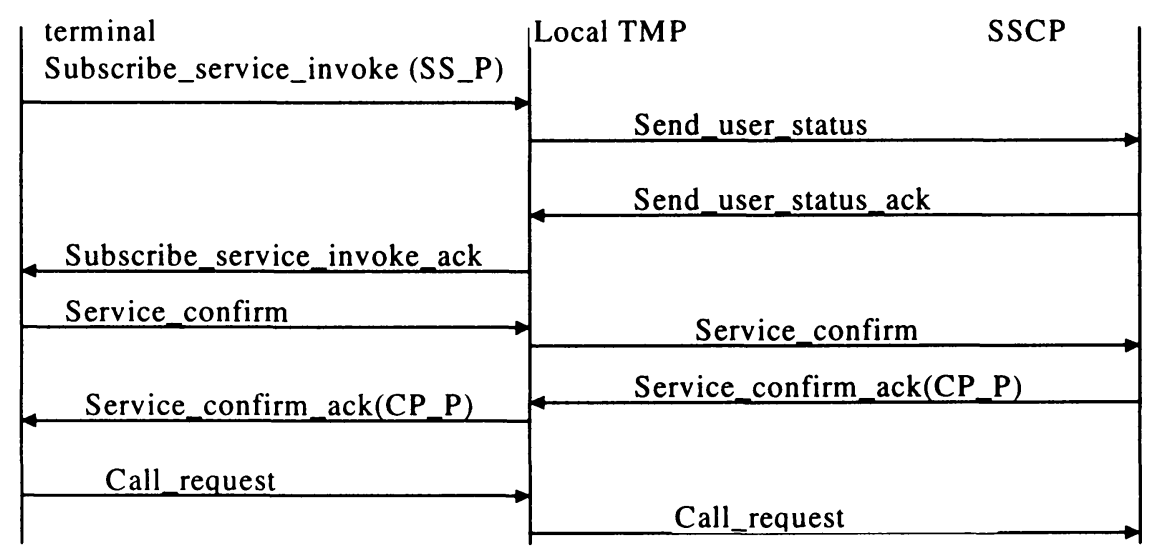

Figure 9 Service invocation

\section{CONCLUSION}

We may conclude from the above considerations that it is possible to develop signaling mechanisms allowing the implementation of a service control architecture that is compatible with the service objectives of TINA-C, which is implementable as an evolution of legacy systems and which takes into account the work of B-ISDN standardization without having to wait for the availability of an adapted and performant Real Time DPE environment. However we have mostly emphasized in this paper the service control aspect of the Broadband Telecommunication. We expect that the signaling mechanisms underlined here, provided that they are complemented with an efficient Call Control signaling will contribute to an efficient Broadband network.

This paper emphasized the signaling requirements at the access network. This allowed us to identify (or make assumptions on) the user and network data that the network infrastructure will have to maintain. In a future paper we will present our current work on how to organize, distribute and maintain the network information model in large scale broadband networks. 


\section{Glossary}

ASF

ASP

B-ISDN

CORBA

DPE

HLR

IE

IN

MAP

OTE

OLEX

SIM

SSCF

SSCP

TEX

TINA-C

Consortium

TLEX

TMF

TMP

TTE

VLR
Access Session Function

Access Session Point

Broadband Integrated Services Digital Network

Common Object Request Broker Architecture

Distributed Processing Environment

Home Location Register

Information Element

Intelligent Network

Mobile Application Part

Originating Terminal Equipment

Originating Local Exchange

Subscriber Identification Module

Service Session Control Function

Service Session Control Point

Transit Exchange

Telecommunication Information Network Architecture

Terminating Local Exchange

Terminal Manager Function

Terminal Manager Point

Terminating Terminal Equipment

Visitor Location Register

\section{References}

C. Rigault, T. Kovacikova: A signaling scheme for B-ISDN and IN integration. IEEE ATM 97 workshop, Lisboa, Portugal

B-ISDN CS3, Signaling requirements. Feb 96 ITU-T Recommendations

IN CS3, ITU-T Recommendations

E. Darmois: TINA: from concept to reality.

Proceedings ICIN 1996, Bordeaux

W.J. Barr, T. Boyd, Y.Inoue: The TINA Initiative

IEEE Communications Magazine, March 1993

H.323, visual telephones systems and equipment for local area networks which provide a non guaranteed quality of service.

Feb 96ITU-T Draft Recommendations

D. C. Schmidt et al: A high performance end system architecture for real time CORBA.

IEEE Communications Magazine, Feb 97 